TITLE:

\title{
Hydrogen emissions from peripheral plasmas in local island divertor and helical divertor configurations
}

\section{AUTHOR(S):}

Iwamae, Atsushi; Sakaue, Atsushi; Neshi, Nobuhiro; Yanagibayashi, Jun; Hasuo, Masahiro; Goto, Motoshi; Morita, Shigeru

\section{CITATION:}

Iwamae, Atsushi ... [et al]. Hydrogen emissions from peripheral plasmas in local island divertor and helical divertor configurations. PHYSICS OF PLASMAS 2010, 17(9): 090701.

\section{ISSUE DATE:}

2010-09

URL:

http://hdl.handle.net/2433/147201

\section{RIGHT:}

Copyright 2010 American Institute of Physics. This article may be downloaded for personal use only. Any other use requires prior permission of the author and the American Institute of Physics. The following article appeared in PHYSICS OF PLASMAS17, 090701 (2010) and may be found at 


\title{
Hydrogen emissions from peripheral plasmas in local island divertor and helical divertor configurations
}

\author{
Atsushi Iwamae, ${ }^{1, a)}$ Atsushi Sakaue, ${ }^{1}$ Nobuhiro Neshi, ${ }^{1}$ Jun Yanagibayashi, ${ }^{1}$ \\ Masahiro Hasuo, ${ }^{1}$ Motoshi Goto, ${ }^{2}$ and Shigeru Morita ${ }^{2}$ \\ ${ }^{1}$ Department of Mechanical Engineering and Science, Kyoto University, Kyoto 606-8501, Japan \\ ${ }^{2}$ National Institute for Fusion Science, Toki, Gifu 509-5292, Japan
}

(Received 7 May 2010; accepted 6 August 2010; published online 30 September 2010)

\begin{abstract}
Superdense core plasmas with a highly peaked electron density profile have been obtained in reduced recycling discharges in the Large Helical Device [O. Motojima et al., Phys. Plasmas 6, 1843 (1999)]. The polarization separation spectroscopy technique has been applied to characterize $\mathrm{H} \alpha$ emissions in the peripheral region of superdense core plasmas. Fitting positions of the high intensity $\mathrm{H} \alpha$ line are located just outside of the last closed flux surface in the local island divertor configuration. In the helical divertor configuration, high intensity emissions are observed around the inner and outer $\mathrm{X}$ points and along the divertor legs. In both configurations the hydrogen atoms have an inward velocity along the lines of sight. (C) 2010 American Institute of Physics.
\end{abstract}

[doi:10.1063/1.3484223]

Understanding of plasma particle and energy confinement is important for toroidal magnetic confinement fusion research in ITER. ${ }^{1,2}$ Various modes to improve confinement have been discovered, i.e., H-mode, ${ }^{3}$ the pellet enhanced performance mode, ${ }^{4}$ internal transport barriers, ${ }^{5-7}$ the edge transport barrier, ${ }^{8}$ the high density H-mode, ${ }^{9}$ and so on. Hydrogen recycling from the peripheral plasma is one of the key issues to control fueling into and pumping from magnetic confined plasmas. The interplay between core plasma and the edge, scrape-off layer, and divertor plasmas is well known. $^{10}$

Two divertor configurations ${ }^{11}$ are employed in the Large Helical Device (LHD). ${ }^{12}$ The helical divertor (HD) is an intrinsic helical double-null divertor. The local island divertor (LID) (Ref. 13) is a variant of the island divertor which utilizes an externally induced $m / n=1 / 1$ island located in the edge region, where $m$ and $n$ are poloidal and toroidal mode numbers, respectively.

Superdense core (SDC) mode ${ }^{14-17}$ has been discovered in LHD plasmas requiring central fueling via repetitive pellet injection. ${ }^{18}$ The central density of $n_{\mathrm{e}} \sim 5 \times 10^{20} \mathrm{~m}^{-3}$ is maintained through the formation of an internal diffusion barrier (IDB) with an extremely high-density gradient. Initially, the IDB-SDC mode was discovered in the LID configuration. Coupled with a relatively high electron temperature, the IDB-SDC modes exhibit the highest degree of performance $\left(n_{0} T_{0} \tau_{\mathrm{E}}=4.4 \times 10^{19} \mathrm{~m}^{-3} \mathrm{keV} \mathrm{s}\right)$ obtained so far in the LHD. ${ }^{14}$ It was found that the IDB-SDC mode also may be obtained in the open HD configuration, provided that the wall pumping works effectively. ${ }^{19}$ During IDB-SDC discharges, a large magnetic axis shift, the Shafranov shift, is observed due to the high plasma pressure in the core region.

In LHD plasmas, the spectral profiles of He I were ob-

\footnotetext{
${ }^{a)}$ Present address: Fusion Research and Development Directorate, Japan Atomic Energy Agency, Naka, Ibaraki 311-0193. Electronic mail: iwamae.atsushi@jaea.go.jp.
}

served with a high resolution spectrometer. ${ }^{20}$ Through Zeeman splitting of the spectra, the locations of He I emission have been identified clearly on a map of the poloidal cross section to be just outside the region of the ergodic layer where the magnetic field line structure is chaotic (see Fig. 1 of Ref. 20). The plasma polarization spectroscopy technique $^{21}$ is applied to identify the locations of $\mathrm{H} \alpha$ emission, hydrogen atomic temperature, and the line of sight (LOS) component of atomic velocity for an equatorial LOS. The polarization separated spectral profiles are interpreted as superpositions of Zeeman profiles for different magnetic field strengths (see Refs. 22 and 23 and references therein).

LHD is a superconducting heliotron device of which the poloidal/toroidal period numbers are $2 / 10$, and the major and averaged minor radii are from $R=3.5 \mathrm{~m}$ to $4.1 \mathrm{~m}$ and $a$ $=0.64 \mathrm{~m}$, respectively. The maximum toroidal magnetic field is $B_{\mathrm{t}} \leq 3 \mathrm{~T}$. The plasma volume is $V_{\mathrm{p}}=30 \mathrm{~m}^{3}$, and it is characterized by the presence of an edge stochastic magnetic field surrounding the core plasma, an "ergodic layer." In this experiment, the magnetic axis position $R_{\mathrm{ax}}$ and the toroidal magnetic field strength $B_{\mathrm{t}}$ were set at $3.75 \mathrm{~m}$ and $-2.64 \mathrm{~T}$, respectively. Negative $B_{\mathrm{t}}$ means that the magnetic field direction is counterclockwise viewed from the top.

The LID head consists of neutralizer plates and a pumping duct (see Fig. 1 of Ref. 15). The LID head is inserted into the island (about $0.2 \mathrm{~m}$ ) in a horizontally elongated cross section where the island is the widest. Charged particles diffusing out from the core region cross the island separatrix and flow along the periphery of the island. After several toroidal turns, the particles reach the outer separatrix of the island where the LID head is located, and strike its backside, through which the particles are neutralized. The last closed flux surface (LCFS) is determined by the inner separatrix of the island. Plasma-surface interaction occurs ideally only at the LID head. The LID head configuration and the pumping system enable high pumping efficiency and impurity control. In discharges with the LID configuration, reductions in the 
TABLE I. LOS location: height $Z$ and axial rotation angle $\alpha$ of the polarization separation optical components.

\begin{tabular}{ccrcc}
\hline \hline & & & \multicolumn{2}{c}{ Instrumental width (FWHM) in pixels } \\
\cline { 4 - 5 } LOS & $\begin{array}{c}Z \\
\text { (m) }\end{array}$ & $\begin{array}{c}\alpha \\
\text { (deg) }\end{array}$ & e-ray & o-ray \\
\hline 1 & 0.495 & 29.7 & 1.79 & 1.76 \\
2 & 0.391 & 29.9 & 1.72 & 1.69 \\
3 & 0.286 & 36.7 & 1.68 & 1.60 \\
4 & 0.182 & 40.7 & 1.66 & 1.67 \\
5 & 0.104 & 45.5 & 1.67 & 1.61 \\
6 & 0.026 & -45.9 & 1.63 & 1.67 \\
7 & -0.104 & 140.7 & 1.72 & 1.71 \\
8 & -0.234 & 49.6 & 1.73 & 1.78 \\
9 & -0.339 & -24.9 & 1.80 & 1.88 \\
10 & -0.443 & -100.3 & 1.92 & 1.95 \\
\hline \hline
\end{tabular}

particle flux and in the heat load to the HD plates as measured by Langmuir probes and thermocouples have been observed.

We observed LHD plasmas sustained mainly by the three neutral beam injections (NBIs). Repetitive pellet injectors were utilized to achieve central fueling. The time evolution of the plasma parameters in a typical multiple-pellet fuel injection discharge with the LID configuration is shown (see Fig. 2 of Ref. 14). The electron density $n_{\mathrm{e}}$ and temperature $T_{\mathrm{e}}$ profiles are measured by means of the Thomson scattering system. Typical profiles of $n_{\mathrm{e}}$ and $T_{\mathrm{e}}$ in the LID configuration and the HD configuration are presented when the plasma stored energy is at a maximum during the IDB-SDC discharge (see Fig. 2 of Ref. 17).

We measured for each shot the polarization separated $\mathrm{H} \alpha$ line profiles for the LID and the HD configurations with NBI heating. Emissions from the hydrogen in the LHD plasma were observed from one of the spectroscopy ports (see Fig. 3 of Ref. 22). We have ten lines of sight each of which equipped with polarization separation optics to cover the poloidal cross section of the plasma.

Table I shows the height $Z$ and the polarization direction angle $\alpha$ of each LOS. The definition of the magnetic field vector $(|B|, \theta, \phi)$ and the polarization components running parallel to the direction of $\alpha$ and perpendicular to $\alpha-90^{\circ}$ are presented (see Fig. 7 of Ref. 22). The exposure time and the repetition rate were $81 \mathrm{~ms}$ and $10 \mathrm{~Hz}$, respectively. The ten LOSs cover the poloidal cross section of the plasma. The cross section of the plasma is elongated in the major radius direction. The magnetic field $\mathbf{B}$ has been determined accurately by the external coil currents. The magnetic field strength $\mathbf{B}$ is saddle shaped and highest near the helical coil (see Fig. 1 of Ref. 22).

$\mathrm{H} \alpha$ line emissions were resolved into two orthogonally polarized components using the polarization separation optical system. We adopted beam splitting Glan-Thompson polarizers except for LOS 6. For LOS 6 we used Glan-Taylor polarizers as shown in Ref. 22. Each of the linearly polarized extraordinary e-rays and ordinary o-rays was focused by a lens onto the input of an optical fiber with a $400 \mu \mathrm{m}$ core diameter. Each fiber was connected to an optical fiber cable a few hundred meters in length to transmit the light to the diagnostics room. The transmitted light was dispersed by an aberration-corrected Czerny-Turner spectrometer ( $f$ $=1.33 \mathrm{~m}$ ) equipped with a 1800 grooves $/ \mathrm{mm}$ grating and recorded with a charge-coupled device $(1024 \times 1024$ of $13 \mu \mathrm{m}^{2}$ pixels). The liner dispersion of the spectrometer was $0.00405 \mathrm{~nm} / \mathrm{pixel}(0.311 \mathrm{~nm} / \mathrm{mm})$ at $\lambda 656.28 \mathrm{~nm}$ in the first order. Nonidentifiable emission lines were found in the observed wavelength regions of the second and third orders. As a wavelength reference we used a Th-Ar hallow cathode lamp. ${ }^{24}$ Until the ninth experimental cycle, the inner wall of the vacuum vessel was covered with stainless steel tiles. Some reflective effects upon the observed spectral profiles may arise especially for the upper and lower LOS. For the middle LOS around the equatorial plane, the reflection effect is expected to be negligible.

Figure 1 depicts the examples of the observed spectra from LOS 5 for plasmas with either a LID or a HD configuration. The exposure time for the LID configuration and the HD configuration was from 1.123 to $1.204 \mathrm{~s}$ and from 1.424 to $1.505 \mathrm{~s}$, respectively, just after each had attained a maximum of plasma stored energy for its respective LID or HD discharge. The observed mutually orthogonal polarization components are plotted with open and filled circles in the upper and lower panels, respectively. In the upper panels, the peaks shift to a longer wavelength. On the other hand, in the lower panels the peaks shift to a shorter wavelength. Concerning the magnetic field direction in the inner and outer regions, these shifts indicate that the $\pi$-light from the outer region is dominant in the upper panels and that the $\pi$-light from the inner region is dominant in the lower panels. We assume that there are two emission locations for each LOS. The locations $R_{\text {in }}$ and $R_{\text {out }}$ are adjustable parameters for the fit related to magnetic configuration. The $\mathrm{H} \alpha$ line consists of 48 allowed and $6 \Delta J$-forbidden fine structure components (18 $\pi$ and $30 \sigma$ allowed, $2 \pi$ and $4 \sigma \Delta J$ forbidden). The corresponding line strengths and line shifts of the fine structure Zeeman components are taken into account in the fitting procedure. The fitted results are plotted with bars beneath the observed spectra. Two temperature components, cold and warm, are assumed at each location. The hot component originating from the charge exchange process cannot be resolved since it has a broad profile compared to the Zeeman splits. The fitted spectra are plotted in black solid curves. The $\pi$ - and $\sigma$-light of the cold and warm temperature components are plotted in red and blue, respectively. Contributions from the inner and outer emission regions are plotted in solid and dashed curves, respectively. The hot charge exchange spectra are plotted in solid orange.

The above fitting procedure is applied to each of ten LOSs. The determined two emission locations, inner and outer points, are plotted for each LOS. Figure 2 shows the results of the fitting. The area of the red circles is proportional to the emission intensity of the cold and warm components. The blue arrows show the velocity of the emitting atoms along the LOS. Concentric ovals indicate the normalized radius $\rho$. The LCFS $(\rho=1.0)$ is plotted in red. In the LID discharge, the $\mathrm{H} \alpha$ intensity is about one-fourth of that for the HD discharge, and the emission is located just outside of the 

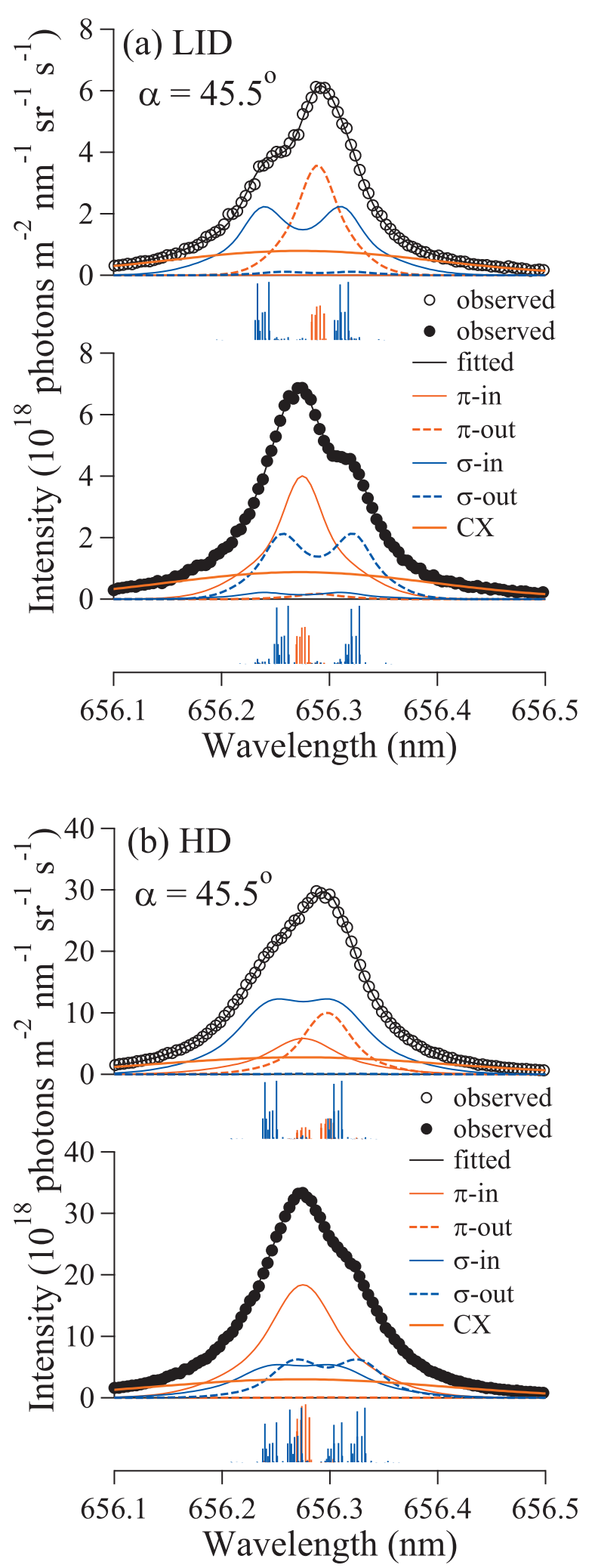

FIG. 1. (Color) Polarization separation spectra observed on LOS 5 for (a) the LID and (b) the HD configurations just after the plasma stored energy attains a maximum during IDB-SDC discharges. N.B. The ordinate scale of LID is $1 / 5$ of HD. Observed mutually orthogonal polarization components are plotted with open and filled circles in the upper and lower panels, respectively. Fine structures are taken into account in the fitting procedure and plotted with bars beneath the observed spectra. The fitted spectra are plotted in black solid curves. The $\pi$ - and $\sigma$-light components are plotted in red and in blue, respectively. Contributions from both the inner and outer emission regions are plotted in solid and in dashed curved lines, respectively. Charge exchange components are plotted in solid orange.
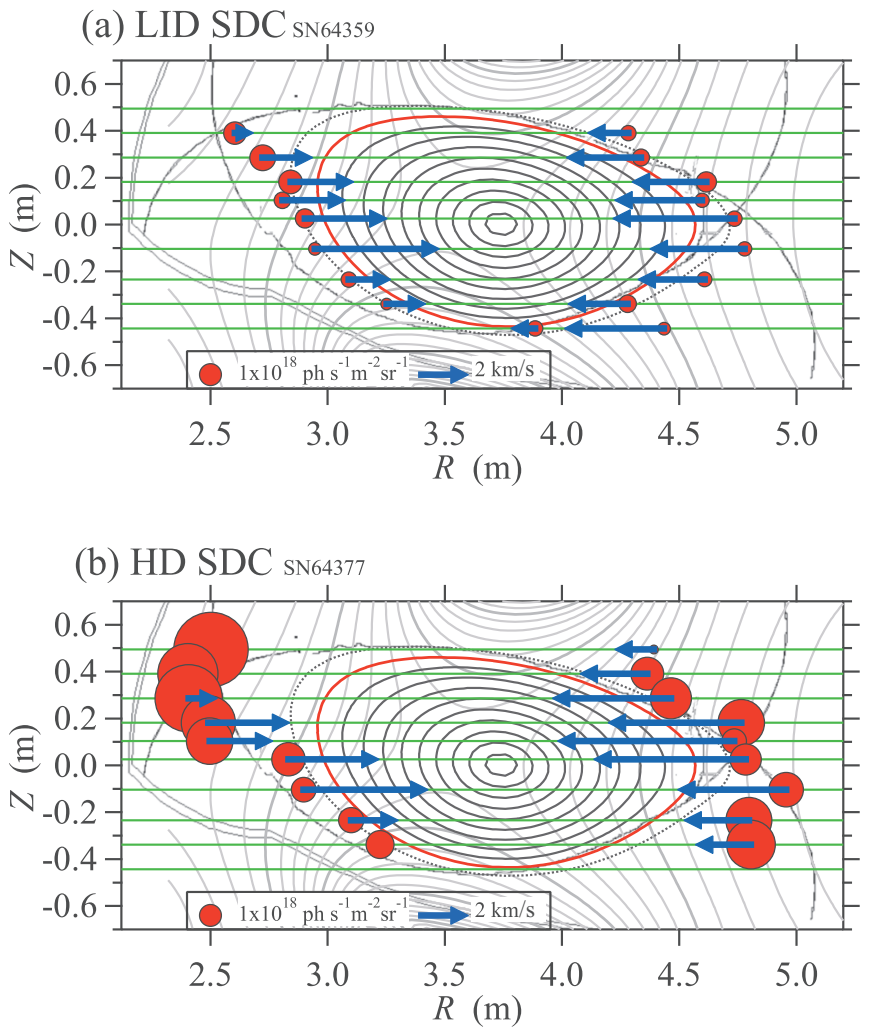

FIG. 2. (Color) Emission intensity distribution along the LOS for (a) the LID configuration and (b) the HD configuration. The area of the red circles is proportional to the emission intensity of a sum of the cold and warm components. The blue arrows show the velocity of the emitting atoms along the LOS.

LCFS. In the HD discharge the emission intensities around the inner X point are rather high and the emissions seem to be located in proximity to the divertor legs. Velocity components along the LOS range from 0.11 to $7.45 \mathrm{~km} / \mathrm{s}$ for the LID configuration and from 0.01 to $7.53 \mathrm{~km} / \mathrm{s}$ for the HD configuration. In the HD configuration the atoms around the inner $\mathrm{X}$ point move a little. On the other hand, the atoms around the outer $\mathrm{X}$ point have a higher velocity along the LOS.

In gas-puff fueled discharge, the density profile is flat and a peaked density profile has not been observed. The LID is capable of pumping out the plasmas in the edge region. It is considered that core fueling with pellet injection and edge particle pumping are key factors to produce the SDC mode.

In the HD discharge after a series of the LID discharges, the SDC mode is observed. It is considered that for low particle load to the first wall and helical divertor plates in the series of LID discharges, wall pumping may be still active in HD-SDC discharge. On the other hand, the observed high $\mathrm{H} \alpha$ light emission at the plasma edge suggests high particle recycling in the HD configuration. Further experimental researches and simulations based on the three-dimensional fluid edge transport code and the kinetic neutral particle transport code are needed to understand quantitative particle confinement in the edge plasma.

The authors are grateful to the LHD experimental group for their support during the course of the experiment and also 
appreciate Super SINET for providing a real-time remote experiment environment. This work is performed with the support and under the auspices of the National Institute for Fusion Science Collaboration Research Program: Grant Nos. L4-323001-007 (FY:H16), NIFS04KOAP010 (FY:H17 and H18), NIFS06KCHP007 (FY:H18), and NIFS07KLPP308 (FY:H19). One of the authors (A.I.) wishes to thank Mr. Tod Tollefson for his careful reading of the manuscript.

${ }^{1}$ F. W. Parkings, D. E. Post, N. A. Uckan et al., Nucl. Fusion 39, 2173 (1999).

${ }^{2}$ M. Shimada, D. J. Campbell, V. Mukhovatov, M. Fujiwara, N. Kirneva, K. Lackner, M. Nagami, V. D. Pustovitov, N. Uckan, J. Wesley, N. Asakura, A. E. Costley, A. J. H. Donné, E. J. Doyle, A. Fasoli, C. Gormezano, Y. Gribov, O. Gruber, T. C. Hender, W. Houlberg, S. Ide, Y. Kamada, A. Leonard, B. Lipschultz, A. Loarte, K. Miyamoto, V. Mukhovatov, T. H. Osborne, A. Polevoi, and A. C. C. Sips, Nucl. Fusion 47, S1 (2007).

${ }^{3}$ F. Wagner, G. Becker, K. Behringer, D. Campbell, A. Eberhagen, W. Engelhardt, G. Fussmann, O. Gehre, J. Gernhardt, G. v. Gierke, G. Haas, M. Huang, F. Karger, M. Keilhacker, O. Klüber, M. Kornherr, K. Lackner, G. Lisitano, G. G. Lister, H. M. Mayer, D. Meisel, E. R. Müller, H. Murmann, H. Niedermeyer, W. Poschenrieder, H. Rapp, H. Röhr, F. Schneider, G. Siller, E. Speth, A. Stäbler, K. H. Steuer, G. Venus, O. Vollmer, and Z. Yü, Phys. Rev. Lett. 49, 1408 (1982).

${ }^{4}$ M. Hugon, B. Ph. van Milligen, P. Smeulders, L. C. Appel, D. V. Bartlett, D. Boucher, A. W. Edwards, L.-G. Eriksson, C. W. Gowers, T. C. Hender, G. Huysmans, J. J. Jacquinot, P. Kupschus, L. Porte, P. H. Rebut, D. F. H. Start, F. Tibone, B. J. D. Tubbing, M. L. Watkins, and W. Zwingmann, Nucl. Fusion 32, 33 (1992).

${ }^{5}$ F. M. Levinton, M. C. Zarnstorff, S. H. Batha, M. Bell, R. E. Bell, R. V. Budny, C. Bush, Z. Chang, E. Fredrickson, A. Janos, J. Manickam, A. Ramsey, S. A. Sabbagh, G. L. Schmidt, E. J. Synakowski, and G. Taylor, Phys. Rev. Lett. 75, 4417 (1995).

${ }^{6}$ E. J. Strait, L. L. Lao, M. E. Mauel, B. W. Rice, T. S. Taylor, K. H. Burrell, M. S. Chu, E. A. Lazarus, T. H. Osborne, S. J. Thompson, and A. D. Turnbull, Phys. Rev. Lett. 75, 4421 (1995).

${ }^{7}$ T. Fujita, T. Hatae, T. Oikawa, S. Takeji, H. Shirai, Y. Koide, S. Ishida, S. Ide, Y. Ishii, T. Ozeki, S. Higashijima, R. Yoshino, Y. Kamada, and Y. Neyatani, Nucl. Fusion 38, 207 (1998).

${ }^{8}$ N. Ohyabu, K. Narihara, H. Funaba, T. Morisaki, S. Masuzaki, K. Kawahata, A. Komori, O. Kaneko, H. Yamada, P. deVries, M. Emoto, M. Goto, Y. Hamada, K. Ida, H. Idei, S. Inagaki, N. Inoue, S. Kado, S. Kubo, R. Kumazawa, T. Minami, J. Miyazawa, S. Morita, S. Murakami, T. Mutoh, S. Muto, Y. Nagayama, Y. Nakamura, H. Nakanishi, K. Nishimura, N. Noda, T. Kobuchi, S. Ohdachi, K. Ohkubo, Y. Oka, M. Osakabe, T. Ozaki, B. J. Peterson, A. Sagara, S. Sakakibara, R. Sakamoto, H. Sasao, M. Sasao, K. Sato, K. Saito, M. Sato, T. Seki, T. Shimozuma, M. Shoji, H. Suzuki, S. Sudo, Y. Takeiri, K. Tanaka, K. Toi, T. Tokuzawa, K. Tsumori, K. Tsuzuki, I. Yamada, S. Yamaguchi, K. Yamazaki, M. Yokoyama, K. Y. Watanabe, T. Watari, and O. Motojima, Phys. Rev. Lett. 84, 103 (2000). ${ }^{9}$ K. McCormick, P. Grigull, R. Burhenn, R. Brakel, H. Ehmler, Y. Feng, R. Fischer, F. Gadelmeier, L. Giannone, D. Hildebrandt, M. Hirsch, E. Holzhauer, R. Jaenicke, J. Kisslinger, T. Klinger, S. Klose, J. P. Knauer, R. König, G. Kühner, H. P. Laqua, D. Naujoks, H. Niedermeyer, E. Pasch, N. Ramasubramanian, N. Rust, F. Sardei, F. Wagner, A. Weller, U. Wenzel, and A. Werner, J. Nucl. Mater. 313-316, 1131 (2003).

${ }^{10}$ P. C. Stangeby, The Plasma Boundary of Magnetic Fusion Device (Institute of Physics, Bristol, 1999).

${ }^{11}$ N. Ohyabu, A. Komori, H. Suzuki, T. Morisaki, S. Masuzaki, H. Funaba, N. Noda, Y. Nakamura, A. Sagara, N. Inoue, R. Sakamoto, S. Inagaki, S. Morita, Y. Takeiri, T. Watanabe, O. Motojima, M. Fujiwara, and A. Iiyoshi, J. Nucl. Mater. 266-269, 302 (1999).

${ }^{12}$ O. Motojima, H. Yamada, A. Komori, N. Ohyabu, K. Kawahata, O. Kaneko, S. Masuzaki, A. Ejiri, M. Emoto, H. Funaba, M. Goto, K. Ida, H. Idei, S. Inagaki, N. Inoue, S. Kado, S. Kubo, R. Kumazawa, T. Minami, J.
Miyazawa, T. Morisaki, S. Morita, S. Murakami, S. Muto, T. Mutoh, Y. Nagayama, Y. Nakamura, H. Nakanishi, K. Narihara, K. Nishimura, N. Noda, T. Kobuchi, S. Ohdachi, Y. Oka, M. Osakabe, T. Ozaki, B. J. Peterson, A. Sagara, S. Sakakibara, R. Sakamoto, H. Sasao, M. Sasao, K. Sato, M. Sato, T. Seki, T. Shimozuma, M. Shoji, H. Suzuki, Y. Takeiri, K. Tanaka, K. Toi, T. Tokuzawa, K. Tsumori, K. Tsuzuki, I. Yamada, S. Yamaguchi, M. Yokoyama, K. Y. Watanabe, T. Watari, Y. Hamada, K. Matsuoka, K. Murai, K. Ohkubo, I. Ohtake, M. Okamoto, S. Satoh, T. Satow, S. Sudo, S. Tanahashi, K. Yamazaki, M. Fujiwara, and A. Iiyoshi, Phys. Plasmas 6, 1843 (1999).

${ }^{13}$ T. Morisaki, S. Masuzaki, A. Komori, N. Ohyabu, M. Kobayashi, Y. Feng, F. Sardei, K. Narihara, K. Tanaka, K. Ida, B. J. Peterson, M. Yoshinuma, N. Ashikawa, M. Emoto, H. Funaba, M. Goto, K. Ikeda, S. Inagaki, O. Kaneko, K. Kawahata, S. Kubo, J. Miyazawa, S. Morita, K. Nagaoka, Y. Nagayama, H. Nakanishi, K. Ohkubo, Y. Oka, M. Osakabe, T. Shimozuma, M. Shoji, Y. Takeiri, S. Sakakibara, R. Sakamoto, K. Sato, K. Toi, K. Tsumori, K. Y. Watanabe, H. Yamada, I. Yamada, Y. Yoshimura, O. Motojima, and LHD Experimental Group, J. Nucl. Mater. 337-339, 154 (2005).

${ }^{14}$ N. Ohyabu, T. Morisaki, S. Masuzaki, R. Sakamoto, M. Kobayashi, J. Miyazawa, M. Shoji, A. Komori, and O. Motojima, and the LHD Experimental Group, Phys. Rev. Lett. 97, 055002 (2006).

${ }^{15}$ S. Masuzaki, T. Morisaki, M. Kobayashi, M. Shoji, N. Ohyabu, A. Komori, and LHD Experimental Group, J. Nucl. Mater. 363-365, 314 (2007).

${ }^{16}$ T. Morisaki, N. Ohyabu, S. Masuzaki, M. Kobayashi, R. Sakamoto, J. Miyazawa, H. Funaba, K. Ida, K. Ikeda, O. Kaneko, S. Morita, S. Mutoh, K. Nagaoka, Y. Nagayama, N. Nakajima, K. Narihara, Y. Oka, M. Osakabe, B. J. Peterson, S. Sakakibara, M. Shoji, Y. Suzuki, Y. Takeiri, N. Tamura, K. Tanaka, K. Tsumori, K. Y. Watanabe, I. Yamada, H. Yamada, A. Komori, and O. Motojima, and the LHD Experimental Group, Phys. Plasmas 14, 056113 (2007).

${ }^{17}$ A. Komori, T. Morisaki, S. Masuzaki, M. Kobayashi, Y. Suzuki, R. Sakamoto, S. Morita, M. B. Chowdhuri, M. Goto, J. Miyazawa, I. Yamada, K. Narihara, N. Ohyabu, A. Sagara, H. Yamada, O. Motojima, and LHD Experimental Group, J. Nucl. Mater. 390-391, 232 (2009).

${ }^{18}$ R. Sakamoto, H. Yamada, K. Tanaka, K. Narihara, S. Morita, S. Sakakibara, S. Masuzaki, S. Inagaki, L. R. Baylor, P. W. Fisher, S. K. Combs, M. J. Gouge, S. Kato, A. Komori, O. Kaneko, N. Ashikawa, P. de Vries, M. Emoto, H. Funaba, M. Goto, K. Ida, H. Idei, K. Ikeda, M. Isobe, S. Kado, K. Kawahata, K. Khlopenkov, S. Kubo, R. Kumazawa, T. Minami, J. Miyazawa, T. Morisaki, S. Murakami, S. Muto, T. Mutoh, Y. Nagayama, Y. Nakamura, H. Nakanishi, K. Nishimura, N. Noda, T. Notake, T. Kobuchi, Y. Liang, S. Ohdachi, N. Ohyabu, Y. Oka, M. Osakabe, T. Ozaki, R. O. Pavlichenko, B. J. Peterson, A. Sagara, K. Saito, H. Sasao, M. Sasao, K. Sato, M. Sato, T. Seki, T. Shimozuma, M. Shoji, S. Sudo, H. Suzuki, M. Takechi, Y. Takeiri, N. Tamura, K. Toi, T. Tokuzawa, Y. Torii, K. Tsumori, I. Yamada, S. Yamaguchi, S. Yamamoto, Y. Yoshimura, K. Y. Watanabe, T. Watari, K. Yamazaki, Y. Hamada, O. Motojima, and M. Fujiwara, Nucl. Fusion 41, 381 (2001).

${ }^{19}$ H. Yamada, R. Sakamoto, J. Miyazawa, M. Kobayashi, T. Morisaki, S. Masuzaki, S. Ohdachi, M. Goto, K. Ida, S. Sakakibara, K. Tanaka, N. Ashikawa, K. Itoh, S. Morita, Y. Nagayama, Y. Narushima, B. J. Peterson, M. Shoji, C. Suzuki, Y. Suzuki, T. Tokuzawa, K. Y. Watanabe, I. Yamada, M. Yokoyama, S. Yoshimura, M. Yoshinuma, S. Imagawa, O. Kaneko, K. Kawahata, T. Mutoh, N. Ohyabu, A. Komori, and O. Motojima, Plasma Phys. Controlled Fusion 49, B487 (2007).

${ }^{20}$ M. Goto and S. Morita, Phys. Rev. E 65, 026401 (2002).

${ }^{21}$ Plasma Polarization Spectroscopy, edited by T. Fujimoto and A. Iwamae (Springer, Berlin, 2008).

${ }^{22}$ A. Iwamae, M. Hayakawa, M. Atake, T. Fujimoto, M. Goto, and S. Morita, Phys. Plasmas 12, 042501 (2005).

${ }^{23}$ A. Iwamae, M. Atake, A. Sakaue, R. Kitai, M. Goto, and S. Morita, Phys. Plasmas 14, 042504 (2007).

${ }^{24}$ D. W. Willmarth, M. Cheselka, M. Fitzpatrick, and M. Rippa, ThoriumArgon Spectral Atlas, http://www.noao.edu/kpno/specatlas/thar/thar.html. 